\title{
Do 'smart' mice feel more pain, or are they just better learners?
}

To THE EDITOR-Whereas Wei and colleagues in a recent Nature Neuroscience article $^{1}$ claim that overexpression of the NMDA receptor subunit NR2B in the forebrain leads to an enhancement of inflammatory pain, the data presented do not necessarily warrant such a claim; instead, the data may suggest that these transgenic mice are better at remembering painful events, and that they learn how to detect certain cues for avoiding potential new injury.

First, these authors did not find any alteration in pain sensitivity and nociceptive threshold in transgenic mice in three types of measurements (tail-flicker test, hot-plate test and cold-plate test). These findings are consistent with data from our laboratory ${ }^{2}$ that these mice exhibited indistinguishable responses to mild foot shock, therefore suggesting that NR2B mice have a normal pain sensitivity and threshold.

Second, the authors examined formalin-injection-induced responses in mice. Injection of 5\% formalin produced indistinguishable responses (licking) in both phase I (initial 10 minutes) and phase II (10-55 minutes), but with more behavioral response at phase III (55-120 minutes). However, judging from their Fig. 6a, it seems that the NR2B transgenic and wild-type mice exhibited a similar degree of residual licking responses in the second half of phase III (between 90-120 minutes). This suggests that the transgenic mice did not feel differently than the wild type mice at this late stage, thus providing unfavorable evidence to the authors' own conclusion that raises doubts about the conclusion that the transgenic mice suffered more inflammatory chronic pain.

Finally, experiments using mechanical non-noxious stimuli may represent a good indication that these transgenic mice are indeed better learners. Typically non-painful, mechanical poking of von Frey fiber to the dorsum of a hind paw elicited no response in animals. However, at one or three days after complete Freund's adjuvant (CFA) injection to the hind paw, wild-type animals responded to the stimulation of the injected (ipsilateral) hind paw, but not much to the contralateral (un-injected) hind paw. With the same protocol, NR2B mice showed $30 \%$ more paw withdrawal responses than wild-type animals in response to stimulation of the injected hind paw. The authors conclude that this is evidence that the mice developed enhanced pain sensitivity.

However, such a conclusion is immediately called into question by one of the authors' additional experiments: NR2B mice also exhibited the same $30 \%$ increase in enhanced paw withdrawal responses to the poking of the contralateral hind paw (un-injected side)! Such a non-specific withdrawal behavior is analogous to a well-known human behavior in which a child who cried after receiving a flu shot a year ago will likely cry once he walks into the doctor's office and sees the syringe and needles. This type of behavioral response is not because the child has developed increased pain sensitivity or feels more pain, but simply reflects the fact that environmental cues re-activate unpleasant memories about the previous painful event; crying (or running out of the clinic) is a defensive behavioral response as a result of memory retrieval. Therefore, the paw withdrawal in response to the mechanical stimulation of the uninjured site strongly suggests that these NR2B mice remembered the bad experience better. Moreover, they are better able to generalize and recognize these cues in order to avoid potential new assaults. To link the non-specific withdrawal responses to the enhancement of pain perception, the authors need to use in vivo recording techniques to show that non-painful poking to the uninjected paw is now capable of eliciting enhanced neural activities in CNS pain perception centers.

A possible effect of learning and memory on paw withdrawal behavior is further supported by the authors' own observation that nociceptive stimuli produced stronger neuronal activation in hippocampal CA 1 and CA 3 regions in the transgenic animals (Fig. 5 of ref. 1). It is well known that the hippocampus is not involved in pain perception, but is a key structure in the formation of memories about people, places and events. The enhanced activation (400\% increase) of hippocampal CA1 and CA 3 cells is consistent with the idea that these transgenic mice produced stronger memories about the occurrence of the injury or insults.
Moreover, our recent study has demonstrated that the activation of NMDA receptors in CA1 neurons is necessary for converting short-term memories into long-term memories ${ }^{3}$.

Therefore, it seems that the behavioral responses reported by Wei and colleagues may be due to the enhanced learning and memory capacity in NR2B mice. Furthermore, their results nicely extended our original finding that these mice are better in learning and memory regardless of whether experiences are pleasant or unpleasant. In fact, remembering bad experiences should be evolutionarily meaningful so that animals can avoid harmful situations or predators, thus increasing their survival.

\section{Yaping Tang, Eiji Shimizu and Joe Z. Tsien}

Department of Molecular Biology, Princeton University, Washington Road, Princeton, New Jersey 08544-1014, USA e-mail: jtsien@princeton.edu

REPLY-Something more than cleverness caused forebrain-targeted NR2B overexpressing mice to respond so robustly to inflammatory stimuli ${ }^{1}$. Tang et al. rightly indicate that transgenic and wild-type mice were no different in tests of acute pain. However, they fail to recognize the significance of a selective enhancement of inflammatory pain in the transgenics. Acute pain relies on intact reflex circuitry, whereas inflammatory pain-induced behavioral responses involve NMDA receptor-mediated synaptic plasticity in central modulatory pathways (for review, see ref. 4). In the latter category, formalin and CFA tests are widely used (for example, see refs. 5-7).

Tang and colleagues' technical concerns about the formalin test are not valid. The fact that phase III responses rose and fell in magnitude during the period of observation (a well-established observation) is irrelevant to the finding that those responses were greatly enhanced in transgenic mice. Furthermore, the demonstration that formalin's effects were dose dependent hardly discredits our study, particularly because formalin-induced central synaptic plasticity depends on the activity level of input fibers ${ }^{8,9}$. The differences between 